\title{
Validação de cenário de simulação realística para ensino do raciocínio diagnóstico na consulta de enfermagem a pacientes com tuberculose pulmonar
}

\author{
Validation of a realistic simulation scenario for teaching diagnostic reasoning in nursing \\ consultation to patients with pulmonary tuberculosis
}

Validación de un escenario de simulación realista para la enseñanza del razonamiento diagnóstico en consulta de enfermería a pacientes con tuberculosis pulmonar

\author{
Maiana Evillyn da Silva Santos \\ ORCID: https://orcid.org/0000-0002-6485-7375 \\ Universidade Federal de Sergipe, Brasil \\ E-mail: maiana_evellyn@hotmail.com \\ Luana Nunes dos Santos \\ ORCID: https://orcid.org/0000-0002-7237-9625 \\ Universidade Federal de Sergipe, Brasil \\ E-mail: luananuneses@gmail.com \\ Joseilze Santos de Andrade \\ ORCID: https://orcid.org/0000-0003-0488-2840 \\ Universidade Federal de Sergipe, Brasil \\ E-mail: joseilzesa@gmail.com \\ José Rodrigo Santos Silva \\ ORCID: https://orcid.org/0000-0002-1918-7122 \\ Universidade Federal de Sergipe, Brasil \\ E-mail: rodrigo.ufs@gmail.com
}

\begin{abstract}
Resumo
A tuberculose ainda persiste como um problema de saúde pública no Brasil e necessita de uma abordagem complexa do enfermeiro para o manejo adequado desta enfermidade. Nesse sentido, o ensino do raciocínio diagnóstico durante a consulta de enfermagem deve ser executado através de metodologias eficazes, dentre elas destaca-se a simulação realista. Objetivo: validar um cenário de simulação realística para o ensino do raciocínio diagnóstico na consulta de enfermagem a pacientes com tuberculose pulmonar. Método: tratou-se de um estudo de desenvolvimento metodológico, realizado no período de janeiro a julho de 2021. O cenário desenhado levou em consideração os itens: design da simulação, determinação de resultados e objetivos, facilitação, briefing, debriefing $e$ avaliação do participante, da simulação e da experiência baseada na simulação. A etapa de validação ocorreu através da aplicação do Índice de Validação de Conteúdo. Adotou-se um nível de significância de 5\% para o teste binomial e 0,80 como índice de proporção de concordância aceitável para validação. Resultados: conforme I-CVI e teste binomial aplicados observouse que todos os itens foram aprovados, com um valor mínimo de 0,88 de proporção de concordância entre os juízes e com uma média de $97 \%$ de aprovação dentre os especialistas. Conclusão: O cenário de simulação realística elaborado e validado para o ensino do raciocínio diagnóstico nas consultas de enfermagem a pacientes com tuberculose pulmonar se caracteriza como uma importante ferramenta metodológica de ensino na formação de estudantes de enfermagem e capacitação de enfermeiros no que tange à assistência a pacientes com tuberculose.
\end{abstract}

Palavras-chave: Ensino; Treinamento por simulação; Educação em enfermagem; Diagnóstico de enfermagem; Tuberculose pulmonar.

\begin{abstract}
Tuberculosis still persists as a public health problem in Brazil and requires a complex approach from the nurse for the proper management of this disease. In this sense, the teaching of diagnostic reasoning during the nursing consultation must be carried out through effective methodologies, among which the realistic simulation. Objective: validate a realistic simulation scenario for teaching diagnostic reasoning in nursing consultations to patients with pulmonary tuberculosis. Method: this was a methodological development study, carried out from January to July 2021. The scenario was designed taking into account the items: simulation design, determination of results and objectives, facilitation, briefing, debriefing and participant evaluation, simulation and simulation-based experience. The validation step took place through the application of the Content Validation Index (IVC). A significance level of 5\% was adopted for the binomial test and 0.80 as an acceptable proportion of agreement index for validation. Results: according to the I-CVI and applied binomial test, it was observed that all items were approved, with a minimum value of 0.88 of agreement proportion
\end{abstract}


among the judges and with an average of $97 \%$ of approval among the experts. Conclusion: The realistic simulation scenario designed and validated for teaching diagnostic reasoning in nursing consultations to patients with pulmonary tuberculosis is characterized as an important methodological teaching tool in the education of nursing students and training of nurses with regard to care for patients with tuberculosis.

Keywords: Teaching; Simulation training; Education nursing; Nursing diagnosis; Tuberculosis, pulmonary.

\section{Resumen}

La tuberculosis aún persiste como un problema de salud pública en Brasil y requiere un abordaje complejo por parte de la enfermera para el manejo adecuado de esta enfermedad. En este sentido, la enseñanza del razonamiento diagnóstico durante la consulta de enfermería debe realizarse mediante metodologías efectivas, entre las que destaca la simulación realista de escenarios. Objetivo: validar un escenario de simulación realista para la enseñanza del razonamiento diagnóstico en las consultas de enfermería a pacientes con tuberculosis pulmonar. Método: se trata de un estudio de desarrollo metodológico, realizado de enero a julio de 2021. El escenario se diseñó teniendo en cuenta los ítems: diseño de simulación, determinación de resultados y objetivos, facilitación incluyendo actividades preparatorias, briefing y entrega de pistas, debriefing y evaluación de los participantes, simulación y experiencia basada en simulación. El paso de validación se llevó a cabo mediante la aplicación del Índice de Validación de Contenido (IVC). Se adoptó un nivel de significancia del 5\% para la prueba binomial y 0,80 como proporción aceptable del índice de concordancia para la validación. Resultados: de acuerdo al I-CVI y prueba binomial aplicada, se observó que todos los ítems fueron aprobados, con un valor mínimo de 0,88 de proporción de acuerdo entre los jueces y con un promedio de $97 \%$ de aprobación entre los expertos. Conclusión: El escenario de simulación realista diseñado y validado para la enseñanza del razonamiento diagnóstico en las consultas de enfermería a pacientes con tuberculosis pulmonar se caracteriza por ser una importante herramienta didáctica metodológica en la formación de estudiantes de enfermería y formación de enfermeras en la atención al paciente con tuberculosis.

Palabras clave: Enseñanza; Entrenamiento simulado; Educación en enfermería; Diagnóstico de enfermería; Tuberculosis pulmonar.

\section{Introdução}

A tuberculose (TB) permanece como uma das doenças infectocontagiosas mais predominantes mundialmente, tendo sido diagnosticados 10 milhões de casos novos no mundo em 2019, além de apresentar uma alta de taxa mortalidade, tendo vitimado 1,4 milhões de pessoas no mesmo ano (Brasil, 2019; World Health Organization, 2020). Embora exista vacina para suas manifestações de maior gravidade, essa enfermidade caracteriza-se como um persistente problema de saúde pública e está associada a determinantes de saúde como condições socioeconômicas precárias, aglomerações populacionais, desnutrição, alcoolismo, dependência química e condições imunossupressoras (da Silva et al., 2018).

O controle de doenças contagiosas no âmbito da saúde pública depende de ações programadas e contínuas, como a busca ativa dos casos, educação em saúde, diagnóstico populacional e epidemiológico, definição dos determinantes e condicionantes de saúde da população e intervenções de promoção e manutenção da saúde (Brasil, 2019; Guimarães, 2017). Diante desse cenário e perante a equipe multiprofissional, enfermeiro exerce um papel fundamental na gestão do cuidado e das ações programáticas de saúde pública, possibilitando assim o diagnóstico precoce da TB e a implementação uma assistência à saúde longitudinal e eficaz ( da Silva et al., 2020; Guimarães, 2017).

$\mathrm{Na}$ assistência de enfermagem, a elaboração de intervenções voltadas ao cuidado do paciente com TB depende da precisão diagnóstica do enfermeiro ao analisar as necessidades do indivíduo, através do exercício de seu raciocínio clínico, o qual é iniciado a partir da graduação ( da Silva et al., 2020). O desenvolvimento do raciocínio diagnóstico do enfermeiro é progressivo e pode ser aperfeiçoado através de diversas metodologias de ensino, dentre elas a simulação realista (SR) de cenários, pois permite ao estudante participar ativamente do processo ensino-aprendizagem ( de Carvalho et al., 2017).

A SR permite aos participantes potencializarem suas experiências cognitivas e psicomotoras através da aplicação dos conhecimentos obtidos na sala de aula em cenários que simulam ambientes clínicos que farão parte do cotidiano profissional (Fabri et al., 2017; Valadares \& Magro, 2014). Além disso, possibilita o desenvolvimento de uma aprendizagem crítica e reflexiva, melhora da atitude e da integração teoria-prática, resultando assim na implementação de uma prática profissional baseada em cuidados seguros através da redução de erros provenientes de condutas inapropriadas (Yamane et al., 2019). 
Um ambiente simulado para obtenção dos objetivos propostos na SR precisa ser adequadamente elaborado, concernindo um aspecto mais próximo possível do ambiente e prática real. Desta forma, é necessário que seja planejado de forma sistemática e intencional, levantando as reais necessidades, com clara definição das metas mensuráveis a serem alcançadas, porém adaptável a realidade que será aplicado ("INACSL Standards of Best Practice", 2016). Para alcance de tal resultado se faz preciso à validação dos cenários e atividades simuladas (Fabri et al., 2017; Tinôco, 2019).

Diante do apresentado, este estudo tem por objetivo validar um cenário de simulação realística para o ensino do raciocínio diagnóstico na consulta de enfermagem a pacientes com tuberculose pulmonar.

\section{Metodologia}

Tratou-se de um estudo de desenvolvimento metodológico para construção e validação de um cenário de simulação realística para o ensino do raciocínio diagnóstico na consulta de enfermagem a pacientes com tuberculose pulmonar, realizado no período de janeiro a julho de 2021, desenvolvido em duas etapas: elaboração do cenário e validação do conteúdo e aparência do cenário (Andrade, 2016). Foram considerados todos os aspectos éticos dispostos na Resolução nº 466/2012 do Conselho Nacional de Saúde do Ministério da Saúde. O projeto foi submetido e aprovado pelo Comitê de Ética em Pesquisa (CEP) da Universidade Federal de Sergipe, sendo aprovado o Parecer nº 4.962.789 e CAAE 31401820.8.0000.5546.

Inicialmente, para a construção do cenário foi realizada uma revisão integrativa da literatura para sintetizar a produção científica sobre diagnósticos de enfermagem para pacientes com tuberculose (Santos et al., 2021) os quais foram utilizados no roteiro do cenário. Em seguida, após leitura e seleção do referencial metodológico ("INACSL Standards of Best Practice”, 2016), realizou-se a elaboração textual do roteiro do cenário. Conforme manual, o cenário foi desenhado levando em consideração os itens: design da simulação, determinação de resultados e objetivos, facilitação incluindo atividades preparatórias, o briefing e a entrega de pistas, debriefing $e$ avaliação do participante, da simulação e da experiência baseada na simulação. Posteriormente foi realizado a validação de conteúdo e aparência por juízes especialistas e a validação da aparência através do público alvo, finalizando com as adequações do cenário a partir das recomendações da validação de conteúdo e da aparência.

Participaram do estudo oito juízes especialistas com comprovada vivência e conhecimento na temática trabalhada e dez discentes de enfermagem, representantes do público alvo, matriculados nos dois últimos períodos do curso de enfermagem da Universidade Federal de Sergipe após assinatura do Termo de Anuência pela coordenadora do curso.

Os critérios estabelecidos para seleção dos juízes especialistas foram adaptados do estudo de Andrade (2016), sendo selecionados juízes cujo perfil era compatível com uma pontuação igual ou superior a dez pontos. Cada titulação recebeu a seguinte pontuação: doutorado em enfermagem e áreas afins - 04 pontos; mestrado em enfermagem e áreas afins - 04 pontos; especialização na área de sistematização da assistência em enfermagem, simulação realística, validação e/ou tuberculose- 02 pontos; dissertação em uma das áreas citadas - 01 ponto; prática assistencial/ docência, mínimo de 02 anos, em uma das áreas citadas- 02 pontos; publicação de pesquisas e/ou livros/capítulos de livros , em uma das áreas citadas - 02 pontos; participação ou orientação em linha de pesquisa e/ou projeto de pesquisa, em uma das áreas citadas - 02 pontos; orientação para doutorado, mestrado ou especialização , em uma das áreas citadas -02 pontos.

A seleção dos juízes foi por conveniência, realizada na Plataforma Lattes do currículo de pesquisadores no portal do Conselho Nacional de Desenvolvimento Científico e Tecnológico ( $\mathrm{CNPq}$ ) utilizando os unitermos da seguinte maneira: (sistematização da assistência de enfermagem, validação ou simulação) e tuberculose; estes foram associados aos filtros disponíveis na plataforma (formação acadêmica/titulação e atuação profissional). Com essa estratégia de busca foram analisados 82 currículos lattes que constavam a titulação de doutorado e 13 currículos lattes com titulação de mestrado.

Levando em consideração a pontuação mínima estabelecida foi enviado convite a 41 doutores e dois mestres, com aceite de quatro doutores. Tomando como referência a recomendação de que o número de juízes seja no mínimo de cinco e máximo de 
dez expertises (N. M. Silva et al., 2019), fez-se necessário a realização de convite à docentes pesquisadores nessa temática, assim foram convidados nove docentes dos quais quatro responderam positivamente, completando a amostra de juízes especialistas do estudo.

A validação da aparência do cenário pelo público alvo foi realizada por alunos matriculados nos dois últimos períodos do curso de enfermagem da Universidade Federal de Sergipe. Os critérios de inclusão dos discentes foram: ter cursado disciplinas em que seja aplicado o Processo de Enfermagem na prática e ter 18 anos ou mais. Dos 22 alunos matriculados, dez aceitaram participar do estudo.

Para coleta de dados, os juízes especialistas foram convidados por meio de uma carta convite. Aos que aceitaram participar foi enviado via e-mail o Termo de Consentimento Livre e Esclarecido (TCLE), o roteiro do cenário de simulação realística, além de três links do Google Forms: instrumento de caracterização dos juízes especialistas, instrumento de validação de conteúdo e aparência adaptado do modelo utilizado por Andrade (2016) e escala de acurácia diagnóstica de enfermagem (EAD) para análise dos diagnósticos de enfermagem sugeridos no roteiro (Matos \& Cruz, 2009). Este artigo apresenta apenas os resultados referentes à validação do cenário.

Aos alunos convidados por meio de carta convite, aqueles que aceitaram participar, foi enviado via e-mail o TCLE, o roteiro do cenário de simulação realística e o link do Google Forms contendo instrumento de caracterização dos discentes e instrumento de validação de aparência. A avaliação realizada pelos juízes especialistas conteve três categorias de domínios: objetivo, estrutura e apresentação e relevância, e a realizada pelo público alvo teve cinco categorias de domínio: objetivos, organização, estilo da escrita, aparência e motivação.

Os itens foram julgados conforme uma escala de Likert, onde foi admitido "Concordo Totalmente"= 1, "Concordo"= 2, "Nem concordo/Nem discordo"= 3, "Discordo"=4, "Discordo totalmente"=5 (SAMPIERI et al., 2013). Desta forma foram considerados aprovados os itens que receberam as notas 1 ou 2. Para validação, utilizou-se o Índice de Validação de Conteúdo (IVC) de três formas (Galindo-Neto et al., 2019). Primeiro foi obtida a concordância dos juízes acerca de cada item, denominado de I-CVI (Item-Level Contente Validity Index); na segunda etapa foi calculada a proporção de itens que obteve concordância de cada juiz, denominada de S-CVI (Scale-level Contente Validity Index); e a terceira etapa consistiu na média do S-CVI, denominada de S-CVI/AVE (Scale-level Contente Validity Index, Averange Calculation Method). O teste binomial, com nível de significância de 5\%, foi utilizado para verificar se a proporção de concordância foi estatisticamente igual ou superior a 0,80 (Polit et al., 2004), sendo assim considerado como desejado na validação. A análise dos dados ocorreu na versão 4.1.0 do software $\mathrm{R}$.

\section{Resultados}

O Quadro 1 apresenta um resumo do roteiro do cenário validado. Estão descritos os itens: nome do cenário, referencial metodológico, objetivos gerais e específicos, resultados esperados, formato da simulação, métodos de avaliação, tempo de duração, materiais necessários, participantes/ atores, ambientação e caracterização dos participantes, caso simulado e debriefing. 
Quadro 1 - Cenário de simulação realística para o raciocínio diagnóstico de enfermagem para pacientes com tuberculose pulmonar.

\section{CENÁRIO DE SIMULAÇÃO REALÍSTICA PARA O RACIOCÍNIO DIAGNÓSTICO DE ENFERMAGEM PARA} PACIENTES COM TUBERCULOSE PULMONAR

\section{Nome do cenário: Raciocínio Diagnóstico de Enfermagem para paciente com Tuberculose Pulmonar}

Referencial metodológico: a elaboração do cenário seguiu as diretrizes da The International Nursing Association for Clinical Simulation and Learning (INACSL) ("INACSL Standards of Best Practice", 2016)

\section{Objetivos:}

Geral: desenvolver o raciocínio diagnóstico de enfermagem para pacientes com tuberculose pulmonar. Específicos: desenvolver o julgamento clínico critico-reflexivo frente ao caso trabalhado e elencar corretamente os Diagnósticos de Enfermagem (DE), segundo a NANDA-I 2018-2020 e/ou da CIPE® 2019/2020, aplicáveis ao caso clínico.

Resultados esperados: Levantamento dos principais possíveis DE relacionados ao caso, com escolha de uma ou ambas taxonomias a seguir:

NANDA-I 2018-2020: padrão respiratório ineficaz, ventilação espontânea prejudicada, troca de gases prejudicada, hipertermia, nutrição desiquilibrada: menor do que as necessidades corporais, intolerância à atividade, fadiga, dor aguda, dor crônica, ansiedade, insônia, distúrbio na identidade pessoal, conhecimento deficiente, comportamento de saúde propenso a risco, manutenção ineficaz da saúde, risco de suicídio, tristeza crônica e sofrimento espiritual.

CIPE® 2019/2020: dispneia moderada, expectoração diminuída, frequência respiratória aumentada, limpeza de vias aéreas diminuída, ritmo respiratório aumentado, tosse aumentada, troca gasosa diminuída, febre real, apetite diminuído, nutrição dietética prejudicada, peso corporal diminuído, intolerância a atividade moderada, fadiga, dor no tórax, baixa autoestima, ansiedade, insônia, angústia espiritual, isolamento social, tristeza crônica, conhecimento em saúde diminuído, abuso de tabaco e abuso de álcool.

Formato da simulação: Clínica simulada de alta fidelidade

\section{Métodos de avaliação:}

$\checkmark$ A avaliação dos participantes será realizada de duas maneiras: levantamento dos DE relacionados ao caso trabalhado na simulação, com posterior comparação dos DE elencados e seus respectivos graus de acurácia diagnóstica validados pelos juízes especialistas e aplicação do instrumento de Inventário Raciocínio Diagnóstico (A. da S. Rodrigues, 2012)

$\checkmark \quad$ A avaliação da simulação será realizada mediante aplicação da Escala de Design da Simulação (Almeida, Mazzo, Martins, Pedersoli, et al., 2015).

$\checkmark$ A avaliação da experiência baseada em simulação será realizada mediante aplicação após adaptação da Escala de Satisfação dos Estudantes e Autoconfiança na Aprendizagem (Almeida, Mazzo, Martins, Baptista, et al., 2015)

\section{Tempo de duração:}

Duração do Briefing: 10-15 minutos

Duração do caso simulado: 10- 15 minutos

Duração do Debriefing: 30-40 minutos

Materiais necessários: para sala de estar- no mínimo cadeiras e/ou sofá; para o quarto- no mínimo cama; prontuário, estetoscópio, esfignomanômetro, oximetro de pulso portátil, termômetro, balança digital, fita métrica, máscara cirúrgica, máscara N95 ou PFF2, óculos de proteção, roupas próprias, jaleco e telefone celular.

Participantes/Atores: Paciente, enfermeira e público alvo da simulação (facilitador/docente e discentes/enfermeiros).

Ambientação e caracterização dos participantes: A cena ocorrerá em um ambiente domiciliar, se sala de estar- presença de cadeiras ou sofá; se quarto- presença de cama. O paciente estará vestido com roupas próprias e em uso de máscara cirúrgica. O profissional enfermeiro estará vestido com roupas próprias e jaleco, em uso da máscara N95 ou PFF2 e óculos de proteção.

\section{Caso simulado (lido aos participantes pelo facilitador antes do início da cena):}

Pedro Guilherme (PG), 26 anos. Nega doenças crônicas. É fumante ativo desde os 17 anos, 3 marços/dia; relata consumo excessivo de bebida alcoólica semanal, possui estilo de vida sedentário e sem acompanhamento médico anterior. Nega alergias medicamentosa e alimentar. Estado civil solteiro, sem filhos, reside sozinho em quarto de vila de aluguel. Concluiu o ensino fundamental (oitava série). Não possui emprego fixo e trabalha como servente de pedreiro.

Semanas anteriores procurou a UBS em busca apenas de medicações que controlassem o estado febril, sendo convidado para consulta e avaliação médica. Mediante consulta, investigação clínica e realização de exames foi diagnosticado com tuberculose pulmonar, por meio de exame bacteriológico - baciloscopia de escarro positiva (BAAR +), além de apresentar imagem radiológica compatível com a infecção com infiltrado e cavidades bilaterais. Foi realizado o teste rápido para HIV, após aconselhamento e consentimento, o qual resultou negativo. Também foram solicitados exames laboratoriais complementares incluindo função hepática, renal e glicemia de jejum. Na semana anterior, foi iniciada a fase intensiva do tratamento para tuberculose, conforme peso na consulta de $56 \mathrm{~kg}$, foi prescrito 4 comprimidos de RHZE (rifampicina, isoniazida, pirazinamida e etambutol).

Maria Eduarda (ME), enfermeira da Unidade Básica de Saúde (UBS) Pedrinhas é acionada pela agente comunitária de saúde da sua área para avaliar Pedro Guilherme que está em Tratamento Diretamente Observado (TDO) para tuberculose em domicílio. A mesma relata que ao supervisionar a tomada da quinta dose diária notou que o paciente não estava bem.

\section{Script da cena (realizada pelos atores):}

A cena inicia com a enfermeira ME chegando ao domicílio paramentada, em uso da máscara N95 ou PFF2 e dos óculos de proteção e abordando o paciente PG de forma empática e cordial:

ME- “- Bom dia, Pedro! Vim hoje te visitar pois soube que você não está se sentindo bem. Como o senhor está?"

Pedro encontra-se sozinho em seu domicílio em uso de uma máscara cirúrgica conforme orientado. Bastante ansioso e com fácies de medo, alternadas com expressões de dor. Estava visivelmente dispneico, com episódios de tosse não expectorativa. Recebe a enfermeira de forma apática:

PG- "- Bom dia. Estou indo.... Não vejo a hora disso tudo acabar! [PAUSA...] Maria, pode me chamar de você mesmo." 
ME- Com voz suave, responde: “- Pedro você se encontra no quinto dia de tratamento. Observei na ficha de supervisão de tomada de medicação que você fez o uso correto durante esses cinco dias. Na semana passada te expliquei que normalmente o tratamento tem duração de 6 meses, sendo os dois primeiros de fase intensiva, em que os medicamentos devem ser ingeridos diariamente e em dose única, sem interromper o tratamento."

Neste momento Pedro acena de forma positiva com a cabeça e a enfermeira prossegue com a fala: “- Por isso, vamos ser otimistas que em breve você retomará a sua vida e seguirá a sua rotina. Volto a te lembrar da importância de seguir corretamente o tratamento. Faz pausa de alguns segundos e continua: "Você está sozinho?"

PG- "Maria, estou sim só. Eu vivo só. Como te falei, moro sozinho neste quartinho alugado e só tenho a minha mãe que mora longe e não tem condições de se deslocar! Falo com ela uma vez ao dia quando a vizinha empresta o aparelho celular, porque infelizmente não temos condições de comprar um para ela." [Fala com olhar de tristeza]. "Por isso preciso melhorar! Preciso voltar a trabalhar para sustentar minha casa e ajudar a minha mãe!" [Fala com tom de ansiedade e tristeza].

Enfermeira notava certo desconforto respiratório durante a fala de Pedro, entrecortada por episódios de tosse seca irritativa.

ME- "Imagino o quanto deve estar sendo difícil para você! Tenho certeza que a sua mãe está bastante preocupada com seu estado de saúde". Faz uma breve pausa e com mudança no tom de voz continua: "Pedro, a quanto tempo está apresentando essa difilcudade para respirar? Quando iniciou todo esse cansaço?"

PG- "Maria, desde a tarde de ontem que não estou me sentindo muito bem! Venho me sentindo muito cansado e com aumento da dor no peito. Essa dor já venho sentindo há mais de três meses, porém piorou bastante nos últimos dias. Meu corpo também começou a doer bastante na última semana. O médico me explicou que poderia ser devido a doença."

ME- "- Além do cansaço e da dor, está sentindo algo mais?"

PG- "- Ontem à noite me senti mais quente e suei muito. Eu não tenho forças suficiente para fazer as coisas de casa e nem cuidar de mim. Passo o dia todo na cama!"

ME- "- Pedro, nesta última semana você vem se alimentando bem? Como está o seu apetite? Como estão sendo as suas refeições?"

PG- "Hoje não consegui comer quase nada, na verdade não venho comendo bem faz tempo e notei que na última semana estou pior. Não sinto fome, mal consigo tomar líquidos, apesar de já saber que devo me esforçar para voltar a comer... Em três meses já perdi mais de 17 quilos".

Após notar o comprometimento alimentar, a enfermeira faz a busca por reações adversas aos fármacos utilizados no tratamento.

ME- "Após o início de tratamento você apresentou algum desses sintomas: enjoos, vômitos, dor no estômago, suor ou urina de cor de cor vermelha, coceiras, manchas pelo corpo, dores de cabeça ou alguma outra coisa?"

PG- Com o olhar vago responde: "- Não não! Não tive nada disso ”

Pedro com ar de tristeza continua:

PG- "Maria, meu problema é outro! [PAUSA]. Minha mente dói, me sinto vazio, estou sem coragem para nada, não sinto felicidade com nada. Venho sem conseguir dormir à noite, vejo os dias clarear! Penso o tempo todo na minha situação, na minha saúde, no quanto errei e não dei importância à minha vida. Estou bastante preocupado com tudo isso, com medo."

Enfermeira volta toda atenção para fala de Pedro e acena com a cabeça de modo compreensível, dando-o segurança para continuar a sua fala.

PG- Com voz trêmula: "Às vezes, me passa na mente uma vontade de sumir, desistir de tudo e apenas descansar. Já pensei várias vezes de como posso fazer isso..."

Enfermeira faz expressão de susto e tenta não demonstrar à Pedro tamanha preocupação naquele momento.

ME- "Pedro, estamos aqui para te ajudar! Não pense desta maneira, estamos te assistindo para que fique bem. Apesar da complexidade do seu quadro, a tuberculose é uma doença que tem cura! Também entendo que tem uma vida difícil". Faz uma pausa e continua: "Você conseguiu se consultar com a psicóloga que te encaminhei na semana passada?"

PG- Pedro acena com a cabeça de forma positiva e diz: "Sim, no outro dia conversei com a psicóloga, contei que em grande parte do tempo me culpo pelo que aconteceu. Além de nunca me cuidar eu também não sabia nada sobre a doença. Mas minha cabeça não está ajudando! Irei tentar melhorar e mudar!"

ME- "Olha, você me disse na semana passada que é católico! Então entregue todo o seu problema e tenha fé para enfrentá-lo! Você precisa ser forte e corajoso para lutar! Precisa se ajudar, o tratamento só irá dar certo se você fizer por onde!"

Pedro esbanja um leve sorriso no rosto ao ver como Maria ficou comovida com sua fala.

ME- Continua sua fala com tom assertivo: "Eu já acionei o serviço social para também te ajudar! Como já conversamos, além da tuberculose, existem outros pontos a serem resolvidos. Em breve a assistente social irá te procurar! Além disso, também já te referenciei ao grupo dos fumantes, em breve você fará o seu tratamento como desejado."

PAUSA DE ALGUNS SEGUNDOS.

ME- "Pedro, preciso realizar seu exame físico agora, você me permite?"

Pedro acena com a cabeça de forma positiva. Enfermeira, de forma cuidadosa, ágil e minuciosa, realiza exame físico crânio-caudal e aferição dos sinais vitais. Os achados são falados pela enfermeira em voz alta na medida que realiza o exame físico.

Foi verificado estado geral comprometido, emagrecimento, mucosa ocular hipocorada, além de precária higienização da cavidade oral. Ausculta cardíaca BRNF em 2T sem sopros. Na ausculta respiratória era possível identificar diminuição dos murmúrios vesiculares e sibilos em bases e terço médio bilateral, com expectoração comprometida, além de dor a palpação à caixa torácica (Pedro faz facie de dor no momento da palpação do tórax), uso da musculatura abdominal durante ciclo respiratório. Região abdominal sem anormalidades, RH+, sem dor à palpação. Extremidades livres de edemas, pele fria, acianótica, enchimento capilar lentificado. Sinais vitais: PA- $180 \mathrm{x} 110 \mathrm{mmHg}$, FC- 128 bpm, FR- 27 irpm, SpO2- 90\% e Tax -38,2. Altura- 1,71m, peso corporal- $53 \mathrm{~kg}$, IMC- 18,13 kg/m².

Com expressão de preocupação, a enfermeira fala:

ME- “- Pedro, seu estado geral está comprometido, seus sinais vitais estão alterados e no momento você precisa de um suporte maior!

PAUSA E RESPIRAÇÃO PROLONGADA DE PEDRO

MG-Com voz enfática: "É preciso te encaminhar a um local de pronto-atendimento! Você não tem condições de ficar aqui dessa maneira!"

PG- Com expressão apreensiva e de medo responde: "Maria, eu estava sentindo que isso iria acontecer comigo! Se é para o meu bem, eu irei! Afinal já errei muito..."

ME- Tocando as mãos de Pedro fala: “- Você vai ficar bem! Será assistido e vai melhorar!”

Maria rapidamente aciona o serviço de remoção de referência pelo telefone celular para encaminhar Pedro. 
A cena encerra com facilitar narrando ao público que Pedro foi removido para um pronto-atendimento.

Debriefing: O debriefing seguirá o guia de orientação: "Três estágios do Debriefing Holístico (Góes \& Jackman, 2020)

Fonte: Autores (2021).

Entre os oito juízes especialistas que validaram o cenário cinco foram do sexo feminino e três do sexo masculino, três possuíam de 25 a 30 anos, dois de 30 a 35 anos, dois com 40 anos e um com mais de 55 anos. Em relação a formação e titulação, seis eram doutores e dois estavam cursando doutorado. Todos os juízes eram docentes do nível superior e também possuíam experiência assistencial em enfermagem, dos quais cinco possuíam de três a cinco anos, dois possuíam sete anos e apenas um com mais de 14 anos de experiência.

Acerca da experiência docente em sistematização da assistência em enfermagem e/ou simulação clínica e/ou validação e/ou tuberculose todos os juízes tinham no mínimo dois anos em ao menos um desses campos. Todos os juízes possuem publicações de pesquisas, livros e/ou capítulos na área estudada e já haviam participado ou orientado projetos de pesquisa na área. Sete juízes realização orientação em graduação, especialização, mestrado e/ou doutorado na área e cinco já haviam ministrados capacitações/cursos sobre a temática.

Entre os dez alunos que validaram a aparência do cenário, sete foram do sexo feminino e os demais do masculino; a faixa etária foi entre 22 a 29 anos. Sobre o período que estavam cursando, seis estavam no nono e quatro no décimo período. Todos já haviam aplicado a SAE e realizado o PE em algum momento na academia e sete possuíam experiência como participante ou público alvo de simulação realística.

As Tabelas 1 e 2 apresentam os resultados da análise dos juízes especialistas e dos alunos respectivamente acerca de cada item julgado. Conforme I-CVI e teste binominal aplicado observa-se que todos os itens foram aprovados, com valor mínimo de 0,88 , pelos dois grupos que fizeram a análise.

Tabela 1: Concordância dos juízes especialistas sobre cada item.

\begin{tabular}{|c|c|c|c|}
\hline Questões & $\mathrm{n}(\%) *$ & I-CVI $* *$ & p-valor*** \\
\hline \multicolumn{4}{|l|}{ Objetivo } \\
\hline O conteúdo está coerente com o objetivo do cenário de simulação realística & $8(100,0 \%)$ & 1,00 & \\
\hline & & & 0,3355 \\
\hline Objetivos de aprendizagem estão claros e concisos & $7(87,5 \%)$ & 0,88 & 0,5964 \\
\hline O conteúdo do cenário facilita o raciocínio diagnóstico & $8(100,0 \%)$ & 1,00 & 0,3355 \\
\hline As informações apresentadas estão cientificamente corretas & $8(100,0 \%)$ & 1,00 & 0,3355 \\
\hline Há uma sequência lógica de conteúdo proposto & $8(100,0 \%)$ & 1,00 & 0,3355 \\
\hline \multicolumn{4}{|l|}{ conseguem abranger bem o conteúdo proposto estudado } \\
\hline $\begin{array}{c}\text { As informações/conteúdos são importantes para a qualidade da assistência } \\
\text { prestada }\end{array}$ & $8(100,0 \%)$ & 1,00 & 0,3355 \\
\hline O objetivo do cenário convida e/ou & $7(87,5 \%)$ & 0,88 & 0,5964 \\
\hline \multicolumn{4}{|l|}{$\begin{array}{l}\text { instiga à mudanças no raciocínio diagnóstico em enfermagem para pacientes com } \\
\text { tuberculose pulmonar }\end{array}$} \\
\hline \multicolumn{4}{|l|}{ Estrutura e apresentação } \\
\hline $\begin{array}{c}\text { O roteiro do cenário é apropriado para os estudantes e profissionais de } \\
\text { enfermagem }\end{array}$ & $8(100,0 \%)$ & 1,00 & 0,3355 \\
\hline A linguagem utilizada é de fácil compreensão ao público & $8(100,0 \%)$ & 1,00 & 0,3355 \\
\hline O cenário possui visual atraente que mantém a atenção dos participantes & $8(100,0 \%)$ & 1,00 & 0,3355 \\
\hline Os dados estão apresentados de maneira estruturada e objetiva & $7(87,5 \%)$ & 0,88 & 0,5964 \\
\hline $\begin{array}{c}\text { A forma de apresentação do cenário contribui para o aprendizado dos } \\
\text { participantes }\end{array}$ & $8(100,0 \%)$ & 1,00 & 0,3355 \\
\hline Detalhes contextuais fornecem pistas com base em resultados desejados & $8(100,0 \%)$ & 1,00 & 0,3355 \\
\hline
\end{tabular}




\begin{tabular}{|c|c|c|c|}
\hline $\begin{array}{c}\text { O perfil do paciente fornece dados suficientes para a realização de um raciocínio } \\
\text { diagnóstico de enfermagem }\end{array}$ & $8(100,0 \%)$ & 1,00 & 0,3355 \\
\hline As páginas ou seções parecem organizadas & $8(100,0 \%)$ & 1,00 & 0,3355 \\
\hline \multicolumn{4}{|l|}{ Relevância } \\
\hline $\begin{array}{c}\text { O cenário permite a transferência de conhecimento e aprendizado em relação ao } \\
\text { no raciocínio diagnóstico em enfermagem para pacientes com tuberculose } \\
\text { pulmonar }\end{array}$ & $8(100,0 \%)$ & 1,00 & 0,3355 \\
\hline O tema retrata aspectos-chave que devem ser reforçados & $8(100,0 \%)$ & 1,00 & 0,3355 \\
\hline $\begin{array}{l}\text { O modelo permite a transferência e generalização do aprendizado a diferentes } \\
\text { contextos }\end{array}$ & $8(100,0 \%)$ & 1,00 & 0,3355 \\
\hline O cenário propõe à construção de conhecimento & $8(100,0 \%)$ & 1,00 & 0,3355 \\
\hline O cenário pode circular no meio científico & $7(87,5 \%)$ & $\mathbf{0 , 8 8}$ & 0,5964 \\
\hline
\end{tabular}

*Percentual de concordância; **Item-Level Contente Validity Index; ***Teste binomial. Fonte: Autores (2021).

Na Tabela 1 observa-se que nenhuma categoria de domínio obteve unanimidade entre os juízes. No domínio "Objetivo" três itens obtiveram valor de 87,5\%, nos domínios "Estrutura e apresentação" e "Relevância" apenas um item não obteve aprovação máxima respectivamente.

Tabela 2: Concordância dos alunos sobre cada item.

\begin{tabular}{|c|c|c|c|}
\hline Questões & $\mathrm{n}(\%) *$ & I-CVI** & p-valor**** \\
\hline \multicolumn{4}{|l|}{ Objetivo } \\
\hline O cenário atende aos objetivos propostos & $10(100,0 \%)$ & 1,00 & 0,2147 \\
\hline $\begin{array}{l}\text { O cenário ajuda na qualidade do aprendizado sobre o raciocínio diagnóstico na } \\
\text { consulta de enfermagem a pacientes com tuberculose pulmonar }\end{array}$ & $10(100,0 \%)$ & 1,00 & 0,2147 \\
\hline O cenário está adequado para ser usado por vocês nesse momento & $10(100,0 \%)$ & 1,00 & 0,2147 \\
\hline \multicolumn{4}{|l|}{ Organização } \\
\hline O título é atraente e indica o conteúdo do cenário & $10(100,0 \%)$ & 1,00 & 0,2147 \\
\hline O tamanho do título e do conteúdo nos tópicos está adequado & $10(100,0 \%)$ & 1,00 & 0,2147 \\
\hline Os tópicos têm sequência lógica & $10(100,0 \%)$ & 1,00 & 0,2147 \\
\hline Há coerência entre os objetivos e o conteúdo do cenário & $10(100,0 \%)$ & 1,00 & 0,2147 \\
\hline \multicolumn{4}{|l|}{ Estilo da escrita } \\
\hline A escrita está clara e objetiva & $10(100,0 \%)$ & 1,00 & 0,2147 \\
\hline O texto é interessante & $10(100,0 \%)$ & 1,00 & 0,2147 \\
\hline O vocabulário é acessível & $10(100,0 \%)$ & 1,00 & 0,2147 \\
\hline Há associação do tema de cada sessão ao texto correspondente & $9(90,0 \%)$ & 0,90 & 0,7516 \\
\hline O estilo da redação corresponde ao nível de conhecimento dos discentes & $10(100,0 \%)$ & 1,00 & 0,2147 \\
\hline \multicolumn{4}{|l|}{ Aparência } \\
\hline As páginas ou seções estão organizadas & $10(100,0 \%)$ & 1,00 & 0,2147 \\
\hline A aparência do cenário está simples e clara & $10(100,0 \%)$ & 1,00 & 0,2147 \\
\hline O conteúdo do cenário é atrativo & $10(100,0 \%)$ & 1,00 & 0,2147 \\
\hline \multicolumn{4}{|l|}{ Motivação } \\
\hline O cenário é apropriado para o perfil dos discentes de graduação & $10(100,0 \%)$ & 1,00 & 0,2147 \\
\hline O conteúdo do cenário se apresentam de forma lógica & $10(100,0 \%)$ & 1,00 & 0,2147 \\
\hline A interação é convidada pelos textos. Sugere ações & $10(100,0 \%)$ & 1,00 & 0,2147 \\
\hline Convida/instiga à mudanças de comportamento e atitude durante a assistência & $10(100,0 \%)$ & 1,00 & 0,2147 \\
\hline O cenário propõe conhecimentos para os discentes & $10(100,0 \%)$ & 1,00 & 0,2147 \\
\hline
\end{tabular}

*Percentual de concordância; **Item-Level Contente Validity Index; ***Teste binomial. Fonte: Autores (2021). 
Na Tabela 2 é visto que apenas o item "Há associação do tema de cada sessão ao texto correspondente" do domínio "Estilo de escrita" não obteve aprovação máxima entre os dez alunos.

Nas Tabelas 3 e 4 observamos quais dos avaliadores foram mais rigorosos. Ao total (S-CVI/AVE), foi observado um percentual de aprovação de $97 \%$ dentre os especialistas e $99,5 \%$ dentre os alunos.

Tabela 3: Proporção de concordância dos juízes especialistas referentes à cada item.

\begin{tabular}{ccc}
\hline Juiz & $\mathbf{n}(\boldsymbol{\%})$ & S-CVI \\
\hline Especialista 1 & 1,00 \\
Especialista 2 & $21(100,0 \%)$ & 1,00 \\
Especialista 3 & $21(100,0 \%)$ & 1,00 \\
Especialista 4 & $21(100,0 \%)$ & 1,00 \\
Especialista 5 & $21(100,0 \%)$ & 1,00 \\
Especialista 6 & $21(100,0 \%)$ & 0,95 \\
Especialista 7 & $20(95,2 \%)$ & 0,81 \\
Especialista 8 & $17(80,9 \%)$ & 1,00 \\
S-CVI/AVE & $21(100,0 \%)$ & 0,970 \\
\hline
\end{tabular}

Fonte: Autores (2021).

Dentre os especialistas (Tabela 3), 6 aprovaram todos os i/ens, 1 não aprovou apenas 1 item e ou outro especialista reprovou 3 itens, dentre 21 avaliados, com o menor percentual de mínimo de 80,9\%.

Tabela 4: Proporção de concordância dos alunos referente à cada item.

\begin{tabular}{ccc}
\hline Aluno & $\mathbf{n}(\%)$ & S-CVI \\
\hline Aluno 1 & $20(100,0 \%)$ & 1,00 \\
Aluno 2 & $20(100,0 \%)$ & 1,00 \\
Aluno 3 & $20(100,0 \%)$ & 1,00 \\
Aluno 4 & $19(95,0 \%)$ & 0,95 \\
Aluno 5 & $20(100,0 \%)$ & 1,00 \\
Aluno 6 & $20(100,0 \%)$ & 1,00 \\
Aluno 7 & $20(100,0 \%)$ & 1,00 \\
Aluno 8 & $20(100,0 \%)$ & 1,00 \\
Aluno 9 & $20(100,0 \%)$ & 1,00 \\
Aluno 10 & $20(100,0 \%)$ & 1,00 \\
S-CVI/AVE & & 0,995 \\
\hline
\end{tabular}

Fonte: Autores (2021).

Observamos na Tabela 4 que dentre os alunos, apenas o aluno 4 não aprovou um dos itens avaliados, e os demais alunos aprovaram todos os 20 itens.

Os diagnósticos de enfermagem citados no resumo do roteiro do cenário (Quadro 1), de ambas as terminologias NANDA e CIPE foram selecionados após aplicação e análise da EAD pelos juízes especialistas. Dessa forma, dentre os diagnósticos sugeridos no processo de validação todos foram mantidos com diferentes graus de acurácia.

\section{Discussão}

O ensino de enfermagem almeja promover a formação de profissionais dotados de um pensamento crítico, reflexivo, humanístico e holístico, uma vez que ser enfermeiro exige habilidades e competências para uma adequada identificação e solução 
de fatores associados ao processo saúde-doença em diversos contextos sociopolíticos, econômicos e culturais (Marran et al., 2015; Rodrigues et al., 2016). Nesse sentido, surge a necessidade de se implementar metodologias de ensino que mais se aproximam da realidade que irá ser vivenciada pelos enfermeiros durante o exercício da profissão (Costa et al., 2018).

Dentre essas metodologias de ensino, destaca-se a SR, pois ela proporciona um ambiente controlado, que permite ao estudante implementar as intervenções de enfermagem antes da vivência em cenário real, sendo assim possível o erro, a repetição e o aprimoramento da sua prática profissional sem danos para o paciente (Bortolato-Major et al., 2018; Fabri et al., 2017; Valadares \& Magro, 2014). A construção deste cenário de SR ocorreu sistematicamente e de forma proposital, traçando-se objetivos a serem alcançados, com metas discutíveis em um ambiente que reproduzirá a realidade da maneira mais verosssímel possível, potencializando assim as experiências teórico-práticas dos estudantes ("INACSL Standards of Best Practice", 2016).

No contexto de assistência à saúde dos indivíduos com TB pulmonar, o profissional enfermeiro se destaca uma vez que seu cuidado é prestado de forma holística, considerando os determinantes de saúde associados a esta enfermidade, além de participar ativamente das ações de educação em saúde no âmbito da atenção primária à saúde (N. C. da Silva et al., 2020; Guimarães, 2017). Para exercer esse cuidado holístico, o enfermeiro necessita trabalhar de forma sistematizada, fazendo uso de um instrumento metodológico chamado Processo de Enfermagem, o qual visa direcionar uma assistência individualizada ao paciente através de cinco etapas, a saber: coleta de dados; diagnóstico; planejamento; implementação e avaliação, sendo a segunda etapa o enfoque deste estudo (Resolução COFEN 358/2009, 2009)

Para definição dos DE para os pacientes com TB pulmonar, o enfermeiro necessita de um raciocínio clínico bem desenvolvido pois a elaboração dos destes compreende processos interpessoais, intelectuais e técnicos que visam o julgamento clínico das respostas do indivíduo, da família ou da comunidade em relação ao processo saúde-doença ou a potenciais problemas de saúde, fornecendo assim os subsídios necessários para a seleção das intervenções de enfermagem e para atingir resultados pelos quais o enfermeiro é responsável (Lunney, 2010; Nanda, 2018)

Na literatura não foi identificado cenário simulado que abordasse a temática desse estudo. Assim, a elaboração de um cenário de SR para o ensino do raciocínio diagnóstico nas consultas de enfermagem a pacientes com tuberculose pulmonar, se faz necessário devido à complexidade desta enfermidade em relação à identificação dos diagnósticos de enfermagem (DE) e à implementação das intervenções de enfermagem para este público, pois trate-se de uma doença infectocontagiosa, de tratamento prolongado, que exige mudanças de hábitos de vida e que ainda possui um estigma histórico de preconceito atrelado ao seu prognóstico (Brasil, 2019; Ferreira et al., 2021). Dessa forma, conforme concordância dos juízes, a construção e validação do cenário poderá subsidiar futuros treinamentos de alunos e enfermeiros.

Cenários simulados criados para a desenvoltura do raciocínio clínico e RDE e estudo sobre aprendizagem e utilização da SAE utilizaram o pressuposto que a utilização da SR como metodologia ativa de ensino gera resultados positivos no processo de ensino-aprendizado, reafirmando que o uso do ambiente simulado permite a possibilidade de erros sem riscos ao paciente e ocorrências de danos, assim é possível exercitar a tomada de decisão clínica sem receio de repercussão negativa na condição de saúde (Antunes et al., 2021; Castro et al., 2021; Tinôco, 2019).

Neste estudo, foram selecionados 41 diagnósticos como possíveis elegíveis durante a aplicação do cenário, sendo 18 diagnósticos pertencentes a linguagem padronizada NANDA e 23 que constam na CIPE (Santos et al., 2021). Diferentemente de outros estudos que abordaram a SR como metodologia para o ensino do raciocínio diagnóstico em consultas de enfermagem Rodrigues, 2017; Rodrigues et al., 2020; da Silva et al., 2021), este estudo viabiliza a utilização de dois sistemas de linguagens padronizadas, aspecto elogiado pelos juízes validadores. Isso pode sugerir que, independentemente do sistema de linguagem padronizado adotado, os DE prioritários relacionados ao paciente com TB pulmonar podem ser identificados através da implementação deste cenário. 
A etapa de validação é recomendada para confirmar a confiabilidade, segurança e aplicabilidade do cenário construído (Fabri et al., 2017) Ressalta-se que a validação do cenário ocorreu de forma virtual, em uma única rodada de avaliação diante dos resultados alcançados com os juízes validadores e os alunos com o IVC acima de 0,8 em todos os itens avaliados. Importante destacar que se algum dos itens tivesse obtido pontuação inferior, haveriam novas rodadas de avaliação até obtenção do valor mínimo adotado. Assim como esse estudo, outros trabalhos semelhantes de validação de cenários também concluíram que para garantia da qualidade da prática simulada e alcance dos objetivos propostos é essencial essa etapa (de Carvalho \& ZemMascarenhas, 2020; Mazzo et al., 2017).

Para validação do conteúdo pelos especialistas foram levados em consideração os critérios de formação e qualificação profissional (Coluci et al., 2015). A elegibilidade dos juízes utilizando-se de critérios que envolveram as áreas de validação, sistematização da assistência de enfermagem, simulação clínica e/ou tuberculose foi fundamental, pois para o desenvolvimento do raciocínio diagnóstico de enfermagem (RDE) na temática o conteúdo do cenário precisa permitir o processo de raciocínio clinico acurado intrinsicamente relacionado ao julgamento diagnóstico (de Carvalho et al., 2017; Tinôco, 2019).

A utilização de alunos como participantes ativos representantes do público alvo no processo metodológico de validação da aparência do cenário, que tem por finalidade validar o conteúdo quanto à clareza, facilidade de leitura, compreensão e forma de apresentação, situação identificada em estudo semelhante (Andrade et al., 2019), levou em consideração que a aparência do cenário precisa motivar a leitura e o aprendizado por parte dos mesmos, de forma que sejam atraídos pelo instrumento educativo.

O teste do cenário é preconizado pelas diretrizes da INACSL, na busca de situações não previstas que possam atrapalhar a aprendizagem e garantir a clareza da descrição do cenário ("INACSL Standards of Best Practice", 2016). Assim, outros estudos deverão ser realizados para teste piloto do cenário validado e apresentado neste estudo. Sugere-se que neste futuro momento, além do desenvolvimento do cenário com atores simulados e público alvo, que seja convidado um integrante do público alvo para exercer o papel do enfermeiro, participando assim de forma ativa deste momento da simulação, conforme sugestão de um juiz especialista e um aluno validador.

\section{Conclusão}

O cenário de simulação realística elaborado e validado para o ensino do raciocínio diagnóstico nas consultas de enfermagem a pacientes com tuberculose pulmonar se caracteriza como uma importante ferramenta metodológica de ensino, visto que se faz necessário que o enfermeiro trace diagnósticos de enfermagem condizentes com o estado do indivíduo, família ou comunidade, para a realização do planejamento e implementação de intervenções de enfermagem, a fim de alcançar resultados compatíveis com as reais necessidades dos pacientes.

Dessa forma, espera-se que a disponibilidade do roteiro validado nesse estudo, aliado à formação de mais profissionais para atuar com essa metodologia ativa de ensino, seja um instrumento facilitador na formação de estudantes de enfermagem e capacitação de enfermeiros no que tange à assistência a pacientes com tuberculose. Futuras pesquisas com realização de teste piloto com estudantes e profissionais de enfermagem poderão ser realizadas a fim de verificar a efetividade da aplicabilidade deste cenário de simulação realística, tendo em vista a escassez de estudos nesta área temática. Utilizar um participante da simulação como próprio enfermeiro do cenário é um aspecto sugerido e que deverá ser testado.

\section{Referências}

Almeida, R. G. dos S., Mazzo, A., Martins, J. C. A., Baptista, R. C. N., Girão, F. B., \& Mendes, I. A. C. (2015). Validation to Portuguese of the Scale of Student Satisfaction and Self-Confidence in Learning. Revista Latino-Americana de Enfermagem, 23(6), 1007-1013. https://doi.org/10.1590/0104-1169.0472.2643

Almeida, R. G. dos S., Mazzo, A., Martins, J. C. A., Pedersoli, C. E., Fumincelli, L., \& Mendes, I. A. C. (2015). Validation for the portuguese language of the simulation design scale. Texto \& Contexto - Enfermagem, 24(4), 934-940. https://doi.org/10.1590/0104-0707201500004570014 
Andrade, P. de O. N. (2016, dezembro 16). Construção e validação do cenário de simulação clínica no manejo da hemorragia pós-parto [MasterThesis]. Universidade Federal de Pernambuco. https://repositorio.ufpe.br/handle/123456789/25985

Andrade, P. de O. N., Oliveira, S. C. de, Morais, S. C. R. V., Guedes, T. G., Melo, G. P. de, \& Linhares, F. M. P. (2019). Validação de cenário de simulação clínica no manejo da hemorragia pós-parto. Revista Brasileira de Enfermagem, 72, 624-631. https://doi.org/10.1590/0034-7167-2018-0065

Antunes, M., Bez, M., Perry, G., \& Jane Soares Carvalho, M. (2021). Raciocínio clínico do estudante de Enfermagem: Análise a partir de um simulador virtual. RENOTE, 18(2), 306-315. https://doi.org/10.22456/1679-1916.110243

Bortolato-Major, C., Arthur, J. P., Mattei, Â. T., Mantovani, M. de F., Felix, J. V. C., \& Boostel, R. (2018). Contribuições da simulação para estudantes de graduação em enfermagem. Revista de Enfermagem UFPE on Line, 12(6), 1751-1762. https://doi.org/10.5205/1981-8963-v12i6a230633p1751-1762-2018

Brasil, M. da S. (2019). Manual de Recomendações para o Controle da Tuberculose no Brasil (2a ed). MS/CGDI.

Carvalho, E. C. de, Oliveira-Kumakura, A. R. de S., \& Morais, S. C. R. V. (2017). Raciocínio clínico em enfermagem: Estratégias de ensino e instrumentos de avaliação. Revista Brasileira de Enfermagem, 70, 662-668. https://doi.org/10.1590/0034-7167-2016-0509

Carvalho, L. R. de, \& Zem-Mascarenhas, S. H. (2020). Construção e validação de um cenário de simulação sobre sepse: Estudo metodológico. Revista da Escola de Enfermagem da USP, 54. https://doi.org/10.1590/S1980-220X2019021603638

Castro, L. das N., Santiago, C. M., Olegário, B. da C. D., Cardoso, J. N., Temperini, H. O., Moraes, A. C. B., \& Nunes, A. S. A. (2021). A simulação realística como ferramenta de aprendizagem para a Sistematização da Assistência de Enfermagem. Research, Society and Development, 10(9), e10110917711e10110917711. https://doi.org/10.33448/rsd-v10i9.17711

Coluci, M. Z. O., Alexandre, N. M. C., \& Milani, D. (2015). Construção de instrumentos de medida na área da saúde. Ciência \& Saúde Coletiva, 20, 925-936. https://doi.org/10.1590/1413-81232015203.04332013

Costa, R. R. de O., Medeiros, S. M. de, Martins, J. C. A., Enders, B. C., Lira, A. L. B. de C., \& Araújo, M. S. de. (2018). A simulação no ensino de enfermagem: Uma análise conceitual. Revista de Enfermagem do Centro-Oeste Mineiro, 8(0), Article 0. https://doi.org/10.19175/recom.v8i0.1928

da Silva, N. C., Lima, E. F., Costa, R. M. F., \& de Araujo, E. E. M. (2020). Tuberculose: Assistência de enfermagem na atenção básica. 6(1).

Fabri, R. P., Mazzo, A., Martins, J. C. A., Miranda, F. B. G., Fumincelli, L., \& Baptista, R. C. N. (2017). Construção de um roteiro teórico-prático para simulação clínica. Rev Esc Enferm USP, 7.

Ferreira, B. C. A., Lima, M. K. C., Barbosa, J. S. dos S., Vianna, T. A., Chícharo, S. C. R., \& Duarte, A. C. da S. (2021). Ação potencial do enfermeiro no enfrentamento ao tratamento da tuberculose na estratégia de saúde da família. Research, Society and Development, 10(8), e19710817375-e19710817375. https://doi.org/10.33448/rsd-v10i8.17375

Galindo-Neto, N. M., Alexandre, A. C. S., Barros, L. M., Sá, G. G. de M., Carvalho, K. M. de, \& Caetano, J. Á. (2019). Creation and validation of an educational video for deaf people about cardiopulmonary resuscitation. Revista Latino-Americana de Enfermagem, 27, e3130. https://doi.org/10.1590/1518-8345.2765.3130

Góes, F. dos S. N. de, \& Jackman, D. (2020). Desenvolvimento de um guia de orientação para instrutores: "Três Estágios do Debriefing holístico". Revista Latino-Americana de Enfermagem, 28. https://doi.org/10.1590/1518-8345.3089.3229

Guimarães, M. H. D. (2017). Tuberculose: Uma Reflexão Sobre o Papel do Enfermeiro na Saúde Pública. Revista Científica Multidisciplinar Núcleo do Conhecimento, 15(02), 54-62.

INACSL Standards of Best Practice: SimulationSM Simulation Design. (2016). Clinical Simulation in Nursing, 12, S5-S12. https://doi.org/10.1016/j.ecns.2016.09.005

Lunney, M. (2010). Use of critical thinking in the diagnostic process. International Journal of Nursing Terminologies and Classifications: The Official Journal of NANDA International, 21(2), 82-88. https://doi.org/10.1111/j.1744-618X.2010.01150.x

Marran, A. L., Lima, P. G., \& Bagnato, M. H. S. (2015). As políticas educacionais e o estágio curricular supervisionado no curso de graduação em enfermagem. Trabalho, Educação e Saúde, 13, 89-108. https://doi.org/10.1590/1981-7746-sip00025

Matos, F. G. de O. A., \& Cruz, D. de A. L. M. da. (2009). Construção de instrumento para avaliar a acurácia diagnóstica. Revista da Escola de Enfermagem da USP, 43, 1088-1097. https://doi.org/10.1590/S0080-62342009000500013

Mazzo, A., Miranda, F. B. G., Meska, M. H. G., Bianchini, A., Bernardes, R. M., \& Pereira, G. A. (2017). Ensino de prevenção e tratamento de lesão por pressão utilizando simulação. Escola Anna Nery, 22. https://doi.org/10.1590/2177-9465-EAN-2017-0182

North American Nursing Diagnosis Association International. (2018). Diagnósticos de enfermagem da NANDA-I: definições e classificações 2018-2020. Artemd.

Polit, D. F., Beck, C. T., \& Hungler, B. P. (2004). Fundamentos de pesquisa em enfermagem: Métodos, avaliação e utilização (5º ed). Artmed Editora.

Resolução COFEN 358/2009. (2009). Cofen - Conselho Federal de Enfermagem. http://www.cofen.gov.br/resoluo-cofen-3582009_4384.html

Rodrigues, A. da S. (2012). Raciocínio diagnóstico de enfermeiros e estudantes de enfermagem [Doutorado em Enfermagem na Saúde do Adulto, Universidade de São Paulo]. https://doi.org/10.11606/T.7.2012.tde-14112012-103847

Rodrigues, C. C. F. M., Carvalho, D. P. de S. R. P., Salvador, P. T. C. de O., Medeiros, S. M. de, Menezes, R. M. de P., Ferreira Júnior, M. A., \& Pereira, V. E. (2016). Ensino inovador de enfermagem a partir da perspectiva das epistemologias do Sul. Escola Anna Nery, 20, 384-389. https://doi.org/10.5935/14148145.20160053 
Research, Society and Development, v. 10, n. 13, e413101321351, 2021

(CC BY 4.0) | ISSN 2525-3409 | DOI: http://dx.doi.org/10.33448/rsd-v10i13.21351

Rodrigues, I. D. C. V. (2017). Simulação realística no processo de ensino-aprendizagem do raciocínio diagnóstico de enfermagem [Universidade do Rio Grande do Norte]. https://repositorio.ufrn.br/bitstream/123456789/23359/1/IellenDantasCamposVerdesRodrigues_TESE.pdf

Rodrigues, I. D. C. V., Ferreira, L. B., Lopes, D. C. L., Menezes, H. F., Rocha, C. C. T., \& da Silva, R. A. R. (2020). Simulação realística: Aproveitamento e benefícios para o ensino-Aprendizagem do raciocínio diagnóstico de enfermagem. artigo, 9(7), 1-17. https://doi.org/10.33448/rsd-v9i7.4338

Sampieri, R. H., Collado, C. F., \& Lucio, M. P. B. (2013). Metodologia da pesquisa (5 ed). Penso.

Santos, M. E. S., Santos, L. N., Mesquita, D. C., Andrade, J. S., Lemos, L. M. D., \& Silva, F. J. C. P. (2021). Diagnósticos de enfermagem para pacientes com tuberculose. Revista Enfermagem Atual In Derme, 95(35), e-021098. https://doi.org/10.31011/reaid-2021-v.95-n.35-art.1111

Silva, A. M. da, Bertoncello, K. C. G., Silva, T. G. da, Amante, L. N., Matos, F. G. de O. A., \& Bellaguarda, M. L. dos R. (2020). Acurácia de diagnósticos de enfermagem: Revisão integrativa. Enfermagem Brasil, 19(2), 167-175. https://doi.org/10.33233/eb.v19i2.3078

Silva, M. B. P. da, Rodrigues, B. S., Barreto, F. M., \& Neto, A. G. da S. (2018). Avaliação das condições socioeconômicas e epidemiológicas dos pacientes com tuberculose no brasil entre 2012 a 2016. 3(2). https://revistas.ufpi.br/index.php/jibi/article/view/7336/4985

Silva, P. C. dos S. da, Medeiros, N. A. da S., Cortes, C. de F., Mendes-Rodrigues, C., Deus, K. G. de, Scalia, L. A. M., Holanda, R. G. de, Holanda, C. C. G. de, \& Araújo, S. A. de. (2021). Construção e validação de um caso clínico para uso em simulação do exame físico do sistema respiratório. Research, Society and Development, 10(6), e48310616023-e48310616023. https://doi.org/10.33448/rsd-v10i6.16023

Silva, N. M., Rosado, S. R., Dos Santos, M. A., \& Sonobe, H. M. (2019). Validação de instrumento de caracterização para pacientes com patologias colorretais. Revista de Enfermagem UFPE on line, 13(4), 960. https://doi.org/10.5205/1981-8963-v13i4a237625p960-965-2019

Tinôco, J. D. de S. (2019). Intervenção educacional para o ensino do raciocínio diagnóstico em enfermagem [Tese de Doutorado, Universidade do Rio Grande do Norte]. https://repositorio.ufrn.br/bitstream/123456789/27746/1/Interven\%c3\%a7\%c3\%a3oeducacionalensino_Tinoco_2019.pdf

Valadares, A. F. M., \& Magro, M. C. da S. (2014). Opinião dos estudantes de enfermagem sobre a simulação realística e o estágio curricular em cenário hospitalar. Acta Paulista de Enfermagem, 27, 138-143. https://doi.org/10.1590/1982-0194201400025

World Health Organization. (2020). Global tuberculosis report 2020. World Health Organization. https://apps.who.int/iris/handle/10665/336069

Yamane, M. T., Machado, V. K., Osternack, K. T., \& Mello, R. G. (2019). Simulação realística como ferramenta de ensino na saúde: Uma revisão integrativa. Espaço para a Saúde - Revista de Saúde Pública do Paraná, 20(1), 87-107. https://doi.org/10.22421/15177130-2019v20n1p87 